\title{
Editorial \\ Emerging Information Technologies for Next Generation Communications and Networks
}

\author{
Byung-Seo Kim $\mathbb{D}$
}

Citation: Kim, B.-S. Emerging Information Technologies for Next Generation Communications and Networks. Appl. Sci. 2021, 11, 812. https://doi.org/10.3390/app 11020812

Received: 8 January 2021 Accepted: 11 January 2021 Published: 16 January 2021

Publisher's Note: MDPI stays neutral with regard to jurisdictional clai$\mathrm{ms}$ in published maps and institutional affiliations.

Copyright: () 2021 by the author. Licensee MDPI, Basel, Switzerland. This article is an open access article distributed under the terms and conditions of the Creative Commons Attribution (CC BY) license (https:// creativecommons.org/licenses/by/ $4.0 /)$.
Department of Software and Communications Engineering, Hongik University, Sejong 30016, Korea; jsnbs@hongik.ac.kr

\section{Introduction}

Our lives can be said to be in an era with information and communication devices, and we are pursuing a hyperconnected society with advanced information and communication devices. This information- and communication-based society is becoming more intelligent through artificial intelligence technologies, and it is becoming possible to deliver and store data more securely and reliably because of distributed encryption techniques such as blockchain. To identify the results of this rapidly changing and developing research on information and communication technologies and to interact with each other, an international academic conference was held-The 8th International Conference on Green and Human Information Technology (ICGHIT 2020).

ICGHIT 2020 was held on 5th-7th February, 2020, in Hanoi, Vietnam. The conference is a unique global conference for researchers, industry professionals, and academics who are interested in the latest developments in green and human information technology. The theme of 2020's conference is "Creative and Hyper X Learning Technology." The latest technologies of X-Learning using neural networks are already pervading our daily life regardless of our recognition. They present substantial challenges and great opportunities at the same time. Centering on this theme, we provide an exciting program: hands-on, experience-based tutorial sessions and special sessions covering research issues and directions with applications from both theoretical and practical viewpoints. The conference will also include plenary sessions, technical sessions, and workshops with special sessions. The topics include, but are not limited to the following: Green information technology, energysaving green computing, green IT convergence and applications communication and IoT communications, networks optical, visual light communication ad hoc, sensor Networks M2M/IoT, ubiquitous computing, computer and network security, wireless and mobile security, internet of things security, applied cryptography, security in big data and cloud computing, multimedia processing, smart media technology, speech and signal processing, computer vision and image processing control and intelligent system automatic control, neural network and fuzzy artificial intelligence, HCI intelligent robotics and transportation, HRI brain science and bioengineering SW / HW design, architecture, development architecture and protocols, sustainable sensor networks, information-centric sensor networks, blockchain-based secure sensor networks, AI-based self-evolving sensor networks, sensor/RFID circuits, design system on a chip (SoC), IC system for communication.

The editorial board of ICGHIT 2020 selected a total of 12 high-quality papers among 132 submissions. The selected papers were required to extend 50\% more from the conference paper and throughout extensive peer review process-including 2 nd and even 3rd revisions-only 6 papers were finally published for this SI. In the next section of this editorial, the published 6 papers are briefly introduced.

\section{Contributions}

A paper titled “Deep Learning Model with Transfer Learning to Infer Personal Preferences in Images" [1] proposes a deep convolutional neural network model with transfer 
learning. The model reflects personal preferences from interdomain databases of images that have a typical visual characteristic. In the proposed model, three public image databases are utilized, which are Fashion-Modified National Institute of Standards and Technology database (MNIST), Labeled Faces in the Wild (LFW), and Indoor Scene Recognition. Those databases include images with atypical visual characteristics in order to train and infer personal visual preferences. A gradient class activation mapping (Grad-CAM) approach is applied to the proposed model, providing explanations about personal visual preference possibilities. In the paper, the effectiveness of transfer learning for incremental preference learning is verified by experiments using interdomain visual datasets with different visual characteristics. Moreover, extensive experiments show that the proposed preference-learning model using transfer learning outperforms a preference model without transfer learning. In terms of the accuracy of preference recognition, the proposed model shows a maximum of about $7.6 \%$ improvement for the LFW database and a maximum of about $9.4 \%$ improvement for the Indoor Scene Recognition database, compared to the model without reflecting transfer learning.

A paper in [2] proposes a new Internet of Things (IoT) architecture and protocols by combining Software-defined networking (SDN) and Named data networking (NDN). IoT is a technology that allows various types of data to be received from various sensing devices and processed to perform activities that are beneficial to life. On the other hand, NDN (a practical implementation technique of Information centric networking) is a newly emerging Internet or networking paradigm that may replace the current Internet architecture operating with IP addresses and that fulfills most of the expectations of the IoT. In addition, SDN is another emerging networking paradigm that is highly capable of managing overall networks efficiently by using centralized controller and transforming complex network architectures into manageable and simple ones. The combination of the SDN controller, NDN protocol and architecture over IoT environment and applications can be lethal in the overall performance of the network. One of the issues in NDN over wireless networks is Broadcast storms because of the flooding nature of NDN's forwarding interest and data packets. The broadcast storm causes network congestions and unnecessary energy consumption. In particular, in IoT networks with massive small communications devices; congestions and wasting of energy are more severe and as a result, they cause unnecessary delays, decrease network performance, and increase the cost and packet delay for important packets. To resolve the aforementioned issues, a paper proposes an energy-efficient, priority-based forwarding (EPF) protocol in SDN-enabled NDN-IoT [2]. The EPF scheme uses an efficient flow management of the SDN controller to control the broadcast storm and efficiently forward the priority-based packets. While a defer timer mechanism is used to prioritized the packet upon its arrival to the node, an energy threshold mechanism is used to control energy consumption and improve overall energy efficiency. The proposed scheme is compared with the conventional flooding mechanisms and geographic interest forwarding method through the extensive simulations using an NdnSim simulator. From the evaluation studies, the proposed protocol outclasses the other comparative methods and produces the best performance results in terms of various metrics such as total number of interests and retransmissions, content retrieval time, total number of priority interests, energy consumption, and network lifetime.

The popularity of wearable devices equipped with a variety of sensors that can measure users' health status and monitor their lifestyles has been increasing. In fact, healthcare service providers have been utilizing these devices as a primary means to collect considerable health data from users. The health data collected via wearable devices are useful for providing healthcare service. However, the indiscriminate collection of an individual's health data raises serious privacy concerns, because the health data obtained by wearable devices contain sensitive and private information related to the wearer's personal health and lifestyle. The authors in [3] propose a method in order to securely preserve the data from the wearable devices. The proposed method leverages local differential privacy, which is a de facto standard for privacy-preserving data processing and aggregation, to 
collect sensitive health data. Particularly, this is to mitigate the error incurred by the perturbation mechanism of location differential privacy, at first. The proposed scheme samples a small amount of salient data that represents the original health data, after which the scheme collects the sampled salient data instead of the entire set of health data. The authors performed extensive experiments and experimental results show that the proposed sampling-based collection scheme achieves significant improvement in the estimated accuracy when compared with straightforward solutions. Furthermore, the experimental results verify that an effective tradeoff between the level of privacy protection and the accuracy of aggregate statistics can be achieved with the proposed approach.

In recent daily life, video has become the most important medium for communication among people. Therefore, reversible data hiding technologies for video, which inserts and extracts data without compromising the original content, have been developed. Therefore, some important and private information can be hidden in the video without damaging the original video in order to be used in the copyright protection and distribution field of video. A paper proposes a practical and genuine reversible data hiding method by adopting a multidimensional histogram shifting scheme on quantized discrete cosine transform (QDCT) coefficients in the H.264/AVC bitstream [4]. The proposed method defines the vacant histogram bins as a set of $\mathrm{n}$-dimensional vectors and finds the optimal vector space, which gives the best performance, in a $4 \times 4$ QDCT block. In addition, the secret message is mapped to the optimal vector space, which is equivalent to embedding the information into the QDCT block. The proposed method is comparatively evaluated in terms of Peak Signal-to-Noise Ratio(PSNR), Payload Per Frame (PFF), and File Increases Per Payload (FPP). The evaluation results show that the efficiency of the proposed hiding method is higher than the other compared five existing methods in terms of PSNR, PFF, and FPP. In addition, the image distortion and maximum payload capacity are measured quite high.

Attackers use a variety of techniques to insert redirection JavaScript in order to lead a user to a malicious webpage, in which a drive-by-download attack is executed. In particular, the redirection JavaScript in the landing site is obfuscated to avoid detection systems. The authors in [5] propose a lightweight detection system based on static analysis to classify the obfuscation type and to promptly detect the obfuscated redirection JavaScript. The proposed model detects the obfuscated redirection JavaScript by converting the JavaScript into an abstract syntax tree (AST). Then, the structure and token information are extracted. Specifically, the paper proposes a lightweight AST to identify the obfuscation type and the revised term frequency-inverse document frequency to efficiently detect the malicious redirection JavaScript. The proposed method enables rapid identification of the obfuscated redirection JavaScript and proactive blocking of the webpages that are used in drive-bydownload attacks. The proposed method is comparatively evaluated with JSUNPACK dynamic analysis system over existing dynamic analysis systems, which are usually employed to analyze obfuscated JavaScript in the real world. The evaluation shows that the proposed system has higher accuracy comparing to that of a dynamic analysis system while requiring significantly less analysis time. It thus offers the best attribute of static analysis.

There is a need to extract meaningful information from big data, classify it into different categories, and predict end-user behavior or emotions. Large amounts of data are generated from various sources such as social media and websites. Text classification is a representative research topic in the field of natural-language processing that categorizes unstructured text data into meaningful categorical classes. The long short-term memory (LSTM) model and the convolutional neural network for sentence classification produce accurate results and have been recently used in various natural-language processing (NLP) tasks. Convolutional neural network (CNN) models use convolutional layers and maximum pooling or max-overtime pooling layers to extract higher-level features, while LSTM models can capture long-term dependencies between word sequences, hence are better used for text classification. However, even with the hybrid approach that leverages the powers of these two deep-learning models, the number of features to remember for 
classification remains huge, hence hindering the training process. A study in [6] proposes an attention-based Bi-LSTM + CNN hybrid model that capitalizes on the advantages of LSTM and CNN with an additional attention mechanism. In the paper, the model is trained using the Internet Movie Database (IMDB) movie review data to evaluate the performance of the proposed model. With the test results, it is shown that the proposed hybrid attention Bi-LSTM + CNN model produces more accurate classification results, as well as higher recall and F1 scores, than individual multilayer perceptron (MLP), CNN or LSTM models as well as the hybrid models.

Funding: This research received no external funding.

Informed Consent Statement: Informed consent was obtained from all subjects involved in the study.

Data Availability Statement: No new data were created or analyzed in this study. Data sharing is not applicable to this article.

Acknowledgments: The author of the submissions has expressed appreciation to the work of the anonymous reviewers and the Applied Science editorial team for their cooperation, suggestions and advice.

Conflicts of Interest: The author declares no conflict of interest.

\section{References}

1. Oh, J.H.; Kim, M.; Ban, S.-W. Deep Learning Model with Transfer Learning to Infer Personal Preferences in Images. Appl. Sci. 2020, 10, 7641. [CrossRef]

2. Tariq, A.; Rehman, R.A.; Kim, B.-S. EPF-An Efficient Forwarding Mechanism in SDN Controller Enabled Named Data IoTs. Appl. Sci. 2020, 10, 7675. [CrossRef]

3. Kim, J.W.; Moon, S.M.; Kang, S.; Jang, B. Effective Privacy-Preserving Collection of Health Data from a User's Wearable Device. Appl. Sci. 2020, 10, 6396. [CrossRef]

4. Kang, J.; Kim, H.; Kang, S. Genuine Reversible Data Hiding Technique for H.264 Bitstream Using Multi-Dimensional Histogram Shifting Technology on QDCT Coefficients. Appl. Sci. 2020, 10, 6410. [CrossRef]

5. Han, K.H.; Hwang, S.O. Lightweight Detection Method of Obfuscated Landing Sites Based on the AST Structure and Tokens. Appl. Sci. 2020, 10, 6116. [CrossRef]

6. Jang, B.; Kim, M.; Harerimana, G.; Kang, S.; Kim, J.W. Bi-LSTM Model to Increase Accuracy in Text Classification: Combining Word2vec CNN and Attention Mechanism. Appl. Sci. 2020, 10, 5841. [CrossRef] 